\title{
Good Gay Men Don’t Get “Messy”: Injecting Drug Use and Gay Community
}

Gary W. Dowsett, David Wain, and Deborah Keys 


\begin{abstract}
This paper reports on an ethnographic exploration of gay men who inject drugs in Melbourne, Australia's second largest city, and demonstrates a further diversification of gay men’s lives, characterized previously as living in a "post AIDS” era (Dowsett 1996a). It suggests that gay community plays a crucial part in some men’s accounts of drug use and shapes their experience of drug injection. Injection remains an abject act, which the gay community is reticent to discuss, with consequences for gay men's health, HIV/AIDS, hepatitis C, sex, and gay community. The reticence positions drug taking (particularly drug injection) as an individual issue and as a violation of practices of self care that results both from gay men’s culture of drug use and from the experience of otherness reported by gay injectors. This paper explores emerging paradoxes for gay men in relation to sex, drugs, relationality, sociality, community, and health.
\end{abstract}

Key words: Injecting drug use, HIV/AIDS, Hepatitis C, disease prevention 


\section{Introduction}

Ever since the advent of HIV/AIDS, particularly in the West, the part played by communities of gay men in the evolution of the pandemic has been a source of speculation and debate. In the early—and subsequently discredited—definition of what was to become AIDS as GRID (or Gay-Related Immune Deficiency), and in various attempts to find cause, for example, in such factors as gay men’s recreational use of amyl nitrites or the fragile anus being unable to withstand infection as might the robust vagina, the connections between gay men and their communities, gay sex, and drug use were confidently welded together in what is actually a complex yet indistinct articulation.

Behavioral science went in search of this articulation in correlations with sexual behavior change, named by the early 1990s variously as community norms, gay community attachment, community diffusion, peer influence, and so on (see Dowsett, 1993). Drug use gets added in this mix in its own ways. Gay communities in Australia and in other countries themselves relied on and configured their early and mostly sustained successes in HIV prevention and support for people living with HIV/AIDS as collective activism and as cultural production, thereby confirming their value as a resource in fighting their epidemics over and above the agency of individual behavioral responsibility (e.g., Watney, 1999). But, the contribution of gay communities is largely assumed, untheorized, and under-researched.

This report concerns itself with one aspect—injecting drug use (IDU) by gay men—of the ways in which a gay community plays out its influence and develops its practices in response of the threats of HIV/AIDS and, more recently in Australia (and elsewhere), of the growing epidemic of hepatitis C. The paper derives from an ongoing investigation that underpins a series of studies over the last eighteen years 
involving the lead author and many colleagues, which have addressed, in part or as well, the ways gay community as a social construct is involved, implicated, configured, deployed, and understood in these epidemics (e.g., Dowsett 1996b, 2003; Dowsett, Bollen, McInnes, Couch, \& Edwards, 2001; Kippax, Connell, Dowsett, \& Crawford, 1993). These studies have all been social science efforts, mostly used qualitative methodologies, and all had a primary focus: understanding HIV prevention and sexual behavior change. However, an interest in gay community, beyond construct and as a material form and resource, as an actor in its own right, has always been an underlying interest as well. Those earlier studies focused mainly on Sydney, Australia's largest city and host to nearly half the cumulative Australian HIV/AIDS case load. Other smaller studies in Adelaide (the capital of South Australia) and Darwin (the capital of the Northern Territory) also followed similar issues, often using comparative research methods (Dowsett, Bollen, et al., 2001; Hurley \& Dowsett, 1994). The study reported on here is the first undertaken by the authors in Melbourne, Australia's second largest city and the capital of the state of Victoria. While driven in particular by a concern with the growing hepatitis $\mathrm{C}$ epidemic, the study also offered an opportunity to get some way inside the Melbourne gay community with a similar interest in studying it in mind.

In this paper, the term gay community, when used without either a definite or indefinite article, refers to a discursive and cultural construct as in "gay community values” or "gay community advocacy.” In this usage, no real, identifiable, or specific community is meant. In contrast, the phrases the gay community and a gay community refer to a specific space and material place, and an identifiable grouping of people, such as the Melbourne gay community, the subject of this paper. When used in the plural, as in gay communities, the various gay communities in Australia (Sydney, 
Melbourne, Cairns, etc.) are what is meant, and the plural is used to note that these communities are not necessarily like each other or homogeneous in their membership, infrastructure, and experience.

\section{HIV/AIDS and Hepatitis C in Australia}

The character of the HIV epidemic in Australia is reasonably well known, as is the country's reputation for developing a well coordinated and quite successful response to the epidemic, guided since 1985 by successive national strategies (Commonwealth of Australia, 1989, 1993, 1996). In summary, the cumulative AIDS case total as of December 31, 2003, was 9,262, of whom 6,349 (69\%) had died. Men accounted for $95 \%$ of total cases, and male-to-male sexual transmission of HIV for $81 \%$ of cases, with a further $5 \%$ attributed to male-to-male sexual contact and IDU (National Centre in HIV Epidemiology \& Clinical Research [NCHECR], 2004). Low levels in other usual transmission categories—-heterosexual contact (7\%), other IDU (3\%)—confirmed an epidemic largely contained within Australia’s identifiable gay communities. The HIV prevalence figures are similar, with adjusted cumulative diagnoses of 20,580 cases, of whom $92 \%$ are male, with $77 \%$ attributed to male-tomale sexual contact and a further $4.2 \%$ to male-to-male sexual contact and IDU (with $11 \%$ for heterosexual contact and 4.3\% for other IDU) (NCHECR, 2004). Incident infections are largely due to male-to-male sexual contact (85\%), and male-to-male sexual contact and IDU (4\%). It is not surprising then that gay communities and HIV/AIDS have become almost synonymous in the last two decades in Australia.

In the midst of the HIV epidemic, another silent epidemic was emerging in Australia, namely of hepatitis C virus (HCV). There were 16,734 new cases of hepatitis C diagnosed in 2001 in Australia, with incidence highest among 20-39 year 
olds (NCHECR, 2002). The cumulative total of Australian cases is 157,000, with just over $79 \%$ chronically infected or suffering serious disease. Injecting drug use accounts for $82 \%$ of incident HCV infections. The issue of co-infection by HIV and HCV is also of concern, and $13.9 \%$ of Australians living with HIV/AIDS are also coinfected with HCV (Grierson, Misson, McDonald, Pitts, \& O’Brien, 2002). We do not know the extent or character of HCV infection among Australian gay men specifically. What we do know so far is that HCV co-infection is high in HIVseropositive gay men (and, as noted above, gay men account for $77 \%$ of cumulative HIV infections). Moreover, even though IDU accounts for only $8.5 \%$ of cumulative HIV infections in Australia (adding the two categories listed above in the HIV/AIDS figures), just under half of these occur among men who report also having had sexual contact with other men (NCHECR, 2002). This may indicate a more complex pattern of transmission and prevalence of HIV and HCV than any single-vector analysis provides. Therefore, hepatitis $\mathrm{C}$ is an issue for gay men's health in addition to HIV/AIDS. Further, those concerned with hepatitis C prevention need to recognize that, even with some uncertainty about the sexual transmission of $\mathrm{HCV}$, hepatitis $\mathrm{C}$ may be related to gay men's recreational use of drugs and to their patterns of injecting drugs in particular, just as we now understand the contribution of gay men’s sexual culture to the Australian pattern of HIV transmission and prevention. Exactly how gay community, as a collective cultural product, influences the spread of hepatitis $\mathrm{C}$ is far from clear and that issue was an inspiration for the study reported on here.

\section{Contextualizing the Gay Communities’ Responses}

Australia's gay communities have weathered the devastation of the HIV epidemic with remarkable resilience, but the longer-term social impact of the disease on their sexual cultures and social lives remains under-recognized (Dowsett \& 
McInnes, 1996b). It is less certain that hepatitis C is firmly on the gay communities' radar as yet. There is evidence indicating that younger gay men do not necessarily see HIV/AIDS as the focus of gay life and that many older gay men no longer see that epidemic as the crisis it once was (Dowsett \& McInnes, 1996a). Also, patterns of risktaking in relation to HIV have changed and a significant upturn in rates of unprotected anal intercourse has occurred since 1996 (Bollen, Edwards, Dowsett, McInnes, \& Couch, 1998; National Centre in HIV Social Research [NCHSR], 2003; Richters, et al., 1997). While gay men’s sexual activities have been closely monitored since the mid-1980s in response to HIV/AIDS, the patterns and extent of gay men's drug use have remained peripheral issues. There was a resurgence in gay community life during the 1990s that resulted, inter alia, in an explosion in gay men attending dance parties in a number of towns and cities. Taking recreational drugs became a recognized part of these events (e.g., Maher, 1999), and even provision for those who injected was a low-key aspect of the preparation, for example, providing safe disposal units for injecting equipment. The extent to which other day-to-day gay events, venues, and commercial establishments provided for gay men's drug injecting is less well known. Little is known also about the relationship between gay men’s sexual and drug-taking practices—it is often just assumed—and, indeed, the evidence is different in various countries (e.g., in the United States (U.S.), see Case, Clatts, Egan, \& Navaraez, 2000; Rhodes, et al., 1998; in Canada, see Myers, et al., [n.d.]; in Australia, see Sharp, et al., 1996). Finally, we know even less about the relationship, if any, between developing a life within a particular gay community and the cultural processes structuring sexual and drug-taking behaviors.

Earlier work (Dowsett, Bollen, et al., 2001) explored social changes in the gay communities' responses to HIV/AIDS through the methodology of comparative 
ethnography. These changes were characterized, in part, as living in a post-AIDS epidemic, a term first coined in 1995 (Dowsett, 1996b). That construct has since been used by others in the U.S. and elsewhere (e.g., Rofes, 1998; National Lesbian and Gay Health Association, 1998). The challenge of post-AIDS was, and is, to capture the diversification of gay men's experiences of, and responses to, the HIV epidemic over the last decade in particular, and to signal that the multiple and evolving standpoints available to gay men in relation to HIV/AIDS require new, innovative public heath and community responses. With post-AIDS as a conceptual backdrop, this study on gay injectors explored what was happening among gay men in terms of drug use in general, and drug injection in particular, within the broader context of changing responses by the various gay communities to the HIV epidemic and emerging concern with the HCV epidemic in Melbourne and in Australia.

The Particular Case of Melbourne’s Gay Community

Research on Australian gay men who inject drugs in relation to the culture of recreational drug use in the gay communities is virtually non-existent. There are some basic descriptive behavioral data on gay men's drug use and drug injection (see below), but there is little analysis of patterns of injection or of subcultural meanings associated with, and providing peer support for, drug use in general or drug injection in particular (however, see Southgate \& Hopwood, 1999). Also, there has been little systematic scrutiny of such drug use for the purposes of developing hepatitis C prevention interventions in the gay community health sector. In the case of Melbourne specifically, even less is known.

Melbourne has $20.7 \%$ of the Australian cumulative AIDS case load (NCHECR, 2002). Twelve percent of Victorians living with HIV are co-infected with HCV (Grierson \& Misson, 2002). Melbourne, with nearly four million people, hosts 
Australia's second largest gay community. It has $88 \%$ of Victoria's AIDS cases, $80 \%$ of which are among gay and homosexually active men (Department of Human Services, 2002). There has been some research on recreational drug use in inner Sydney’s gay community (Ireland, Knox, Van de Ven, Howard, \& Kippax, 1999; Southgate \& Hopwood, 1999), but no comparable work had been done in Melbourne (see Benton \& Kirkby, 1994, for an early contribution). Previous behavioral monitoring of Melbourne’s gay community revealed that gay men there did inject in smaller numbers than in Sydney, 5.9\% vs. 11.7\% (Prestage, et al., 1997). However, the most recent figures comparing these two cities vary considerably according to study design: $6.2 \%$ vs. $14.3 \%$ for gay community-attached men in a national 2002 postal survey; $4.8 \%$ vs. $6.4 \%$ in the 2002 gay community periodic behavioral surveys (NCHSR, 2003 p. 59).

That said, assessing the extent of drug injection by gay men, or even the incidence and prevalence of HCV (or HIV) infection in any specific gay community, is not the central issue when it comes to developing educational strategies to deal with hepatitis $\mathrm{C}$ prevention. What is needed is a keener understanding of the social specificities that structure gay men's use of recreational drugs and where drug injection fits into a gay community. A key question for this project then was whether there is a specifically gay subcultural form of drug injection in Melbourne.

Can we generalize for the case of Melbourne’s gay community to other gay communities in Australia or elsewhere? We are not certain, for we do not subscribe to the notion that gay communities are the same the world over. Certainly, our argument here notes that there are specificities that are part of Melbourne's gay community. So, does each gay community have a specific subcultural form of drug use injection? If what we report here makes sense, then that is distinctly possible. Clearly, research 
similar to this study done elsewhere might help us determine the answer to that question. But, in this type of research, it is only at the level of theory and social process that we can suggest generalizable ideas. Hopefully, we do present such ideas here that can be taken up by others and tested elsewhere.

\section{Methodology}

The methodology selected for the kind of cultural and educational inquiry used in this study was critical ethnography (Dowsett, 1996b), an approach that focuses on cultural forms and processes rather than on individual behavior, and in this case that is also informed by theories of gay community and sexuality. The study was very much an example of what Burawoy et al. (1991) term ethnography as extended case method. The ethnography comprised individual and group interviews, community observations, and a concurrent gay media analysis. The individual interviews were designed to investigate the life histories of gay men and the evolution of their sexual interests and community participation, as well as their drug-taking careers and preferences, in order to situate drug-taking behavior in a broader, more social and contextual frame. The group interviews were designed to explore, inter alia, the relationship between drug use and interests that grow from being gay within the context of daily gay community life. The community observations were focused on providing more certainty to available anecdotal evidence about the sites for drugtaking in the gay scene, that is, its social, sexual, commercial, and cultural sites, and in events and circumstances that constitute gay culture as a set of interrelated daily practices. The gay media analysis added another layer to these observations.

\section{Study Respondents}

Thirty individual focused interviews were conducted with a small number of key informants and with gay men who inject drugs. The respondents were recruited 
by advertisements in gay community newspapers, through printed flyers left in gay venues, clinics, and services, and through health education programs in local HIV/AIDS services networks. These flyers and advertisements took various forms, including small cards wrapped in plastic Ziplock packets commonly used for carrying recreational drugs. Men were asked to telephone a number at La Trobe University where the study was conducted.

A theoretical sampling process was used (Strauss \& Corbin, 1990), that is, we started with men who volunteered in response to these first recruitment strategies, who injected at the time of the study, who regarded themselves as gay, and who resided in Melbourne (not in nearby commuter towns or in non-metropolitan areas), and interviewing proceeded. The interviews occurred in tandem with preliminary analysis so that further respondents could be selected based on data already retrieved, gaps could be noted, and issues that were emerging or reaching saturation could be identified (Strauss \& Corbin, 1990). The recruitment process was monitored by logging certain characteristics of the respondents as the fieldwork progressed to assist further recruitment that would ensure a sample both as typical and as heterogeneous as possible within the study definitions. These characteristics were age, HIV and/or hepatitis $\mathrm{C}$ infection status, and drug of choice. Particular attention was paid to spotting any sample skewing that might result from the small payment of AU\$20 offered for participation (a less usual research practice in Australia than in the U.S.), and at one point, it was clear that more heroin than amphetamine users had been attracted to the study. Consequently, subsequent interview places were then largely reserved for amphetamine users, which led to some fieldwork timetable issues. While steroid injectors had not been specifically excluded from the study design, they proved particularly difficult to find, and were eventually excluded from the study. 


\section{Field Methods}

Individual interviews were conducted by the second author at the host research center, the Australian Research Centre in Sex, Health and Society, at the participating community-based HIV/AIDS agency (see below), or in respondents' homes. The interviews were guided by a focused interview question schedule, flexibly followed by the interviewer to produce a conversational feel. The interviews documented: (a) existing patterns of recreational drug use; (b) the specific pattern of injecting drug use; (c) initiation into drug injection; (d) social relations and practices (parties, venues for socializing, injection sites, and contexts); (e) understandings of HCV transmission; (f) patterns of purchasing injection equipment and of use (sharing, sterilization, exchange, etc.); (g) gay sexual relations during which drug injection occurred; (h) meanings of drug use and of injection in particular; (i) use of services (AIDS agencies, IDU organizations, needle and syringe programs, etc.); (j) gay community opinions about, and expectations in relation to, drug use and injecting drug use in particular.

Four in-depth group interviews, each consisting of between six and eight gay men, were conducted by the second author and the research assistants with men recruited with the assistance of the participating community-based HIV/AIDS agency and through similar community advertisements. The groups were composed of, respectively: (a) HCV seropositive and HCV seronegative gay men who injected; (b) HIV seropositive gay men who injected; (c) gay men under twenty-six who used illicit drugs but did not inject; (d) gay men over thirty who used illicit drugs but did not inject. A fifth group of gay educators who had developed HCV prevention programs was later added to obtain an assessment of Melbourne-based educators’ knowledge and experience of hepatitis $\mathrm{C}$ and drug use issues as a starting point for potential 
training programs (assessed nationally more systematically in Dowsett, Turney, Woolcock, Rance, \& Thomson, 1999; Dowsett, Misson, McDonnell, \& McInnes, 2001). These group interview categories were suggested by the participating community-based HIV/AIDS agency, which was concerned with infection status differences in the gay community and their potential effects on community efforts to prevent hepatitis C and HIV infection. These group interviews followed a discussion guide focused on a range of issues and were intended to explore and highlight through discussion between respondents the community aspects of gay men's drug use. One of the great advantages of group interviews is that the circumstances in common among respondents actually produce both convergence and divergence in their assessment of various issues, such as gay community attitudes toward drug use and the operation of sex and drugs in the gay scene. All quotations presented below, unless followed by a citation, are from respondents in these individual or group interviews.

A number of community observations were also undertaken by various members of the research team and volunteers from the participating community-based HIV/AIDS agency over a six-month period at major gay venues in Melbourne (discos, dance parties, pubs, clubs, and community events) to document the positioning of drug injection within the larger network of social relations of gay men. Some of these observations were conducted by research team members alone. On larger occasions (e.g., during Melbourne’s annual, month-long, gay Midsumma Festival at its Fair Day and at Red Raw, that festival's large dance party), service provider staff from the participating community-based HIV/AIDS agency supplemented the team. These were all passive observations; no interaction with respondents or participation in these events and venues occurred as we did not wish to endanger the research team or volunteer observers by accidental or assumed involvement in illicit drug-taking acts or 
associated sexual events, nor did we want their presence to alter events that were attended in any appreciable way.

Finally, during the field work period, a regular scanning by the research team of gay community media and similar sources (community newsletters, magazines, radio programs, and educational resources) followed community discussions or debates, if any, about drug use, and about drug injection in particular, as one way of mapping change in community values and of registering untoward events that might have had an impact on the study (e.g., a police raid of a dance party or sex club).

The project was funded in late 1999; its fieldwork took place in 2000; and a series of community feedback activities were conducted in 2001. The key gay community-based HIV/AIDS agency collaborating in the study was the Victorian AIDS Council/Gay Men’s Health Centre (hereafter VAC), which declined the title of co-researcher or co-investigator, asking instead to be called the community partner, noting that they were not researchers and had different capacities and skills to bring to the collaboration. Ethics approval was granted by the human ethics committees of both La Trobe University and VAC.

Analysis

The critical ethnography focused the analysis on the nature and meaning of drug-taking and -injecting experience and discourse as reported by the respondents and/or noted in the observations and media monitoring. While the individual respondents constituted the sample and formed the initial unit of analysis, the research object was cultural patterning and process, rather than aggregated individual experience. Therefore, case study writing provided the initial analytical technique for the individual interview material, and a thematic analysis drove the interrogation of the group interview material, the community observations, and the media watch. The 
main analysis focused on synthesizing these initial case studies to look at social and historical processes that had helped to develop the drug-using culture of these gay men and to inspire their use of injectable drugs in certain contexts. In addition, a twoday workshop undertaken in conjunction with VAC allowed service provider perspectives on the data and experience to be fed into the analysis. Finally, the study utilized a community advisory group, composed of service providers, policy makers, and researchers, that was facilitated by the host research center's Community Liaison and Education Unit. This advisory group offered early responses to the findings, which further helped to strengthen the study's conclusions.

The kind of research material obtained in such ethnographic studies relies heavily on self-report, memory, interpretation, and skilled analysis. Such studies are not intended to find facts per se; rather, their purpose is to search for information about the meaning-making work people do as they live their lives and negotiate their social worlds. Perception and sense-making decisions or assessments are documented in order to understand how, in this case, gay men who inject drugs see themselves in relation to other gay men and to the gay community, and in the context of their material, sexual, and recreational interests. It is recognized as well that the research process itself is part and parcel of the generation of the very stories and accounts that are studied. Indeed, such moments for some respondents are new experiences, the first time such stories are told and listened to. Some respondents are well practiced in telling their stories (see Plummer, 1995, for an excellent theoretical and cultural account of this narrative process); others find it more difficult. The result is a very rich and complex set of accounts and stories, with justifications, recuperations, and sometimes, even resolutions. 
Also, research findings from critical ethnography studies are interpretive and contingent on the research team's skills, experiences, and disciplinary perspectives. Indeed, the make-up of the team was important. For example, the PI and project coordinator were (still are) both out gay men, as were (are) the two research assistants, both recruited from gay community-based AIDS service organizations. In addition, all four had extensive experience in HIV/AIDS work (e.g., in activism, advocacy, research, politics, prevention education, outreach, and training). The research assistants also had experience in needle and syringe programs and outreach to sex workers (important as it turned out, especially because some of the respondents were sex workers). The PI had a long prior experience in researching gay community responses to HIV/AIDS in a number of gay communities in Australia and abroad, and his more recent research on hepatitis $\mathrm{C}$ prevention provided further background to this study (Dowsett, et al. 1999). The research team was a situated one (Angrosino \& Mays de Pérez, 2000), with extended personal and professional experience of these subcultures in one way or another, a reflexive disposition toward gay men's health, politics, and social issues, and a long experience in HIV/AIDS, thereby bringing to the material obtained empirically in this study an analytical framework based on lengthy, deep, and varied experience of gay community life in Australia. We were, as Race (2003, p. 371) describes it, “scientifically and homosexually active” men.

\section{Findings and Discussion}

This study provided a very rich and complex data set, even though it had only been intended as an exploratory or pilot study due the small level of funding available in the call for research applications that funded it, and as the first study of this kind the research team had been able to do of Melbourne’s gay community. This paper concentrates on one key aspect of the findings: the relationship between gay men who 
inject drugs and the gay community in Melbourne. Additional results were reported to VAC for program development purposes, including those related to: patterns of drug use; drug experiences, including initiation; drugs chosen and their reported effects on respondents; drug use in relation to sex; utilization of services, including equipment provision programs; knowledge of hepatitis C risks and prevention strategies; and potential for new HIV/HCV prevention strategies for gay men who inject drugs.

\section{Gay Community}

Assessing the perceived contribution of Melbourne's gay community to recreational drug use and drug injection presented theoretical issues as well as empirical challenges. This project did not conceptualize gay community as mere context, in the sense of social or cultural backdrop or historical mise en scène. Rather, it was theorized as a construct, a shifting field of play, manufactured daily by the actions and in the impressions of its participants in deliberate and less direct ways through social practices (including sexual ones). It was also viewed as manifesting in the structure of, and resources producing, patterns of association among gay men, or what we call sociality, for example, the way the gay scene organizes social relations and values certain activities; and it was experienced on a day-to-day basis through the character and quality of interpersonal relationships, which we termed relationality, as expressed, for example, through friendship circles, housemates, boyfriends, and life partners. In other words, we saw gay community as providing density, texture, and specificity, as well as possibility and constraint, and as creating an active environment or climate that is produced through and in turn produces individual and collective agency. Consequently, gay community is a physical, emotional, and symbolic space, both practice and discourse at one and the same time. In this way, Melbourne's gay community acts as a specific case of the more general construct gay community. 
Respondents in this study were asked what meaning gay community held for them, and the general consensus was “not much.” Initial reactions were often scornful of the whole idea of gay community; and, indeed, doubts were expressed whether it even existed. Yet it soon became apparent that there were social practices and institutional effects of gay community operating in the men's lives in ways that were so taken for granted that they did not appear worthy of comment. Identifying as a gay man held some value, even if that category was less than firm. In contrast to the surety implied in the gay, lesbian, bisexual, and transgender alphabet soup descriptor, GLBT, some respondents regarded with ambivalence the idea that these subgroups were part of the gay community at all. Still others rejected "gay” for "queer," thereby distancing themselves from a community they saw as aging, HIV/AIDS-affected, and out of touch. Overall, there was a perception that gay men were too diverse in their beliefs, values, and experiences to form a cohesive community, and once this diversity was taken into account, the only common element remaining was, as one respondent noted, “.... network of, um, people with, um, a lot of different backgrounds, a lot of different ideas, um; but like [laughter] butt-fucking each other.”

The gay community, then, was something out there that one could go into, take what one needed from, and leave, satisfied when one had had enough. That, at least, was the expectation. The experience was often different, as we shall see, in that gay men in the study also sought to fulfill their needs through relationships among smaller networks of people within the larger community. Thus, no matter what ambivalence or values concerning gay community were expressed, it was clear that gay community mattered, and it did so in many, often contradictory, ways. As one respondent said: 
Community, I don't know, the word just doesn't even sound right to me, 'cause community, sort of, says everyone's together...if you're not sort of-I don't know-if you're not sort of wearing a red hat today then you're not part of our community...I've never really gone into it in a big way because it's never meant that much.

Melbourne’s gay community and its commercial gay scene were usually differentiated, but then conflated. The scene (mainly consisting of bars, sex clubs, gay agencies, media resources, and specific events) was reported as difficult, as if there were some sort of rule book about how to be gay available somewhere that some of the men had never obtained. Difficulties with fitting in, and with learning the particular and changing social conventions and styles that serve as indicators of belonging to gay community were reported as well. In other words, being uncomfortable in the scene, for example, was sometimes conflated with issues of belonging to gay community. Such notions were noticeably strong among those men in this study who reported problematic patterns of drug use. They often hoped that their needs would be fulfilled by or through gay community, both as an ideal and a material resource, something no community could ever hope to achieve. This discrepancy resulted in bitterness and cynicism among such men.

Melbourne’s gay community (like many others in the West) had gone through a period of significant growth within the lifetimes of some of the study's respondents. One result has been a changing dynamic regarding how a gay community offers increased potential to build new ways of living a gay life. Gay sociality has become more complex, densely textured, and deeply embedded. Yet, something has also been lost in this transformation from the historically hidden homosexual subculture to the modern gay community after gay liberation (see Dowsett, 1996b, for a discussion of 
the distinction). Whatever the gains, the increased diversity and complexity have their downside. For some men in this study, gay community attachment (Kippax, Connell, Dowsett, \& Crawford, 1993) was an ongoing process, never a complete achievement, in which they engaged with mixed success. The principal difficulty for them appeared to be their fear of rejection. Respondents stated that there are specific, expected, and approved, and by inference disapproved, ways for gay men to behave within the gay community. They reported, therefore, that other gay men assess how well one “does gay” (Dowsett, 2003, p. 243) and, as is the case in any such evaluation, there is a chance that one will not pass muster, not be gay in the right way, including with respect to one's body and appearance, and to one’s comportment socially and sexually—and also, it seems, in relation to drug use.

The following excerpt is from a respondent who formerly had a network of gay friends that had been disrupted by a lover's death and by a period of heavy heroin use. He saw this as a temporary, if dispiriting, phase of his life and had plans to rejoin that network:

Hopefully, once I get myself better and stuff, and I get back in...groups that I can, sort of, get in to make friends in the gay scene and stuff. I had a look in the [local gay newspaper] that came out last week for their support—not support groups, but like the friend[ship] groups that get together and do things like go to the movies or go out or have dinner and stuff like that. But, I'm just so scared about making the first step...There's a volley ball team...I've been dying to go and join...or just to go and have a look and see what it's about, but I just feel inadequate. I feel as though I'm not gonna be accepted or I'm too skinny or I look yucky or-and people are gonna judge me all the time. 
For such men, there is, clearly, hard work to be done in "doing gay." Particularly for those in this study who were already troubled by their sexuality and/or their drug use, risking rejection by immersion in gay life was sometimes too large a price to pay. Yet, once this process of forming and deepening relationships was underway, it became easier as men's networks grew. This helps both in increasing the number of people known and in becoming more adept socially. And it was through these networks that respondents often reported that recreational drug use was first learned.

Drug Use and Gay Community

Through this study, we learned that gay men do not encounter drugs free of context, but through their relationships with others, and often with other gay men. Within Melbourne’s gay community, there are clearly networks that involve recreational drug use. To some extent, it is through such networks that we were able to recruit our sample. These networks provided a context for using and access to the substances themselves, that is, they provided for gay men an available version of why and how to use drugs, and therein men learned the choreography of using drugs and of accessing drug-facilitated events (Southgate \& Hopwood, 1999). This social nature of using offered one way to understand drug use as a collective or communal production, or a social practice that was embedded in relationships and not just an individual behavior.

As gay community has changed over time, so too have the attitudes toward and the cultural mores surrounding drugs changed in the broader population, in which drug use has become more widespread and drugs themselves, particularly party drugs, have become more easily available. Our respondents reported that gay men were more 
likely to encounter drugs at a younger age and that drugs were more readily available than in the past:

...younger gay men, younger boys, they're growing up in a culture which is oriented towards drug-taking, so it's not a shock. It's almost like a norm in a sense. So, I think there is a generational thing that depends [on] what generation we're going to talk about...[younger] people have grown up with that and I think that people are likely to accept that. And the availability, like I said, the availability of drugs now is, I think, it's increased a lot more.

Intimate friendship circles and broader social networks are obvious candidates as the contextual factors to which behavioral science might ascribe importance in influencing sexual and drug-taking activity. We saw these as more than mere background but rather as a collective construction that provided the engine and the fuel for doing gay. What was less clear is how gay community life shaped the specific use of drugs and its place within gay culture.

The Drug Experience and "Ways of Using"

One key to understanding gay men’s drug use lies in looking at how they talk about experiences facilitated by drugs, rather than in focusing on the particular drugs used. Study respondents' experiences fell into two broad categories that aligned roughly with the ideas of drug use as "hobby" and as "habit" — terms respondents actually used. The hobby category involved experiences that were expressions of desires to take the user towards a temporary enhancement of everyday life, as described in the following response by a respondent:

Why gay men take drugs is the same kind of reasons that others take drugs, I think. In terms of the recreational level, I suppose whatever you like-it could be a whole range of things like about celebrating sexuality, celebrating a 
culture...I think that, you know, that's a part of exploring boundaries, you know, the next step, the next kind of greater pleasure, enabling pleasure, even about disinhibiting people to enjoy their sexuality. It could be about exploring new limits of their sexuality. It could be a whole range of things.

A number of factors, such as place, company, and time, combined to create a type of experience that is configured by a number of social conventions. Hence, pleasure was reported as being a product of where, when, and how the drugs were used, of the special lighting, music, and venues involved, and of the company of likeminded people, all coming together to create a positive experience. The hobby category then was aligned with pleasurable use of drugs in gay social settings, and respondents contrasted this hobby basis for drug use with a second category of use described as a type of habit. Habit use consisted in experiences that expressed needs to take the user away from something problematic and to sustain these experiences as long and as frequently as possible. One key informant explained it thus:

Most people use their drugs - the central elements of music, crowd, and drug are part of the same experience, and that's everybody out there doing the same thing together to interact. And, the drugs, while not the only part of it, are certainly the integral part of it. And people use that—it's basically an enhancement...It's not, they're not coping or using it to cope with some other issue. It's there for its own sake. Whereas, I guess, people with a behavioral or chemical addiction to these things are using it because they can't stop, or because they don't want to. So, they're doing it because- -they don't want to--because there are other issues there that aren't being sorted out and aren't being resolved and aren’t being addressed. So that's why they do it, as an escape. 
This distinction was also a functional one used by gay men to distinguish between specific gay men and their different patterns of using. This occurred not just at the interpersonal level between friends or others in any given friendship circle or social network, but also suffused the Melbourne gay community’s way of framing certain kinds of drug use as somewhat beyond the pale, something abject, even when the practice was not uncommon. And respondents argued that the injection of drugs should be placed beyond the pale.

Similar to other Western cities with large gay communities, Melbourne’s drugfacilitated experience was assigned a particular space at the community-wide level. This space was centered on the phenomenon of (largely) gay dance parties and, to a lesser extent, dance clubs. Indeed, men in this study reported that being a regular in this scene often included learning about drugs in these settings. Yet, it was possible to attend such events in Melbourne and not be aware of drug use because of a high level of discretion surrounding its use. Study respondents reported this as an unquestioned part of the acceptable use of drugs, ensuring that strangers (even in the context of a gay dance party) would never observe the actual administration of the drug:

Code words are still used for drugs. Um, and even when code words aren’t used, it, it, the drugs are hinted at but not talked about or not mentioned. Like, oh, they're "naughty" and that kind of stuff, and it's framed as in "being naughty,” like with a smile on your face; and I, ah, I find that's bizarre. Indeed, as part of this study, during a community observation undertaken at the annual Red Raw dance party, there was no formal acknowledgement of drug use, despite the large number of discarded, empty Ziplock bags (used for storing drugs) littering the dance floor. Drug use was to that extent visible, even if actual administration was not; but injecting of drugs was almost completely invisible. 
Indeed, the count of needles and syringes in the disposal units after Red Raw was under ten, with no other injection equipment discarded thoughtlessly elsewhere. The provision of such disposal units at these events is not uncommon in a country that has had free, nationally funded needle and syringe programs since the 1980s, unlike the U.S.; but these programs are the result of prudent public health strategies, not of widespread cultural approval for injecting drugs. The lack of discarded injecting equipment suggests that injection was largely happening elsewhere (in car parks, at home, before the event, etc.), rather than as something integrated into the overall ebb and flow of other, discrete, non-injected drug-taking at such events (as indicated by the discarded Ziplock bags).

This particular discretion concerning injection also had an impact on this study. We were informed that there may be some men-mostly middle-class gay men who injected drugs — who would not participate in this study for fear of exposure of their drug use, despite having been informed of our strict human subjects ethics processes. One consequence was that many study respondents came from workingclass backgrounds and were more likely to be regular heroin injectors (who veered closer to a habit pattern of use) than occasional amphetamine injectors (who would be closer to a hobby pattern). However, because there are no definitive class-based assessments available to understand the constitution of Melbourne's gay community (nor of any other gay communities world-wide for that matter), in an exploratory qualitative study of this kind we resisted any notion that our sample represented the general drug-injecting population of gay men in Melbourne, and instead attempted to redress the recruitment imbalance in part through key informant interviews recruited within the theoretical sampling process described earlier. Nonetheless, we were convinced that this ethic of discretion prevented some gay men who injected, at least 
recreationally, from participating. It is also possible that this community dynamic could have important consequences for developing community-based hepatitis C prevention education, in that such men might not best be reached through group participation programs or activities that rely on their physical presence and consequent exposure as injectors to other gay men not in their circle of friends.

\section{Initiation}

Starting to use drugs is a complex business. Respondents reported that their initiation into drug use had come about in two basic ways: either by growing up in a drug-using milieu (neighborhood, family, schools) or by encountering drug use later in life. Broadly speaking, among our respondents, the former related more to a habit pattern of drug use and to a more tenuous connection to gay community, while the latter was more common among the hobby users, who also had a stronger attachment to gay community (for related findings on the relational character and social contexts of initiation, see Crofts, Louie, Rosenthal, \& Jolley, 1996). Growing up in drug-using milieus before forming a strong sense of being gay was suggested by some of our respondents as producing patterns of drug use different from those perceived as occurring within the larger gay community, particularly within what such respondents saw as more middle-class lifestyles. Sensing this difference was more often linked to coming from working-class backgrounds and, while this pattern could have resulted from a study sampling effect, it was clearly reported by such men. Forming a strong attachment to gay community—described not just as "being gay” but also as “doing gay”- was complicated by this sense of dissociation from gay community.

The second pattern involved learning gay community “approved” forms of using drugs within a stronger, pre-existing attachment to gay community life and tended to include men from backgrounds other than working class. Again, we caution 
about our sampling; but there was a noticeable contrast between these men and those who had established patterns of drug use before coming into contact with a gay community. Gay community-based drug use initiation and experience enhanced a sense of community, serving as a mark of membership and confirming status as an insider:

My first E [ecstasy] was actually done to go to a party. And I remember the big...routine we went through. Oh, this is your E, you know. It was like, oh, wow! Ha! And because we... all took them together-and it was like hugs, kisses, and let's go in and have the best time. And it was a real marvelous way, ah, to do it.

Satisfactory drug experiences can lead to a desire to learn more about drugs and to seek new experiences. The most common way this occurred in our study was reported to be through contact with more experienced users who had found ways to organize the use of drugs as an ongoing part of their lives. This we refer to as ways of using — that is, meanings associated with, and patterns of, using drugs that utilized the various choreographies of using specific drugs and of experiencing drug-facilitated events, which grew from the qualities of sociality and relationality that occurred within a gay community (see also Sharp et al., 1991, for an early definition of "ways of using," which did not refer to techniques of drug administration, but rather to the sociocultural underpinnings of using).

Similar bonding undoubtedly occurs in other drug-using populations or circles. What is important to note about gay ways of using drugs is that the bond between users also reflects ways of doing gay, of living gay lives, and of being part of a marginalized and stigmatized subculture and an identifiable community in which sexual interests are the defining characteristic. That is not necessarily true for other 
injectors. Indeed, heterosexual drug injectors do not use their drug injection to help constitute and specifically define their sexual orientation.

Respondents reported that encountering more experienced gay users and becoming close enough to them to learn more about drug use also assisted in building stronger attachments to community life. This was a mutually constitutive process, that is, specifically gay ways of using were experienced, and these both were the product of and then helped to produce a distinct gay sociality. Taking on gay ways of using also resulted in drawing individuals who had not come from drug using backgrounds into drug-using milieus, which in turn brought contact with suppliers, who made drugs more accessible, which led to increased use, and brought men into even more contact with drugs and dealers, and so forth. The end result of this process was the increasing movement of the men in this sample into a milieu in which drug use was normalized, if not normative:

I was into the marijuana then, and I had people actually coming to me, purchasing marijuana which then linked me into people. Oh, do you know anyone who’s got some amphetamines? Do you know anyone who’s got ecstasy? Do you know if anyone’s got acid? And it’s always, I suppose, the circle grew out of some of those people-because they had access to certain drugs.

While this pattern of drug uptake is not specific to gay men, we argue that drug use gains a particular character and thereby becomes, for some gay men, part and parcel of doing gay socially and sexually, of forming an attachment to a gay community specifically, and of developing that pervasive sense of belonging that many gay men pursue. 
The recreational consumption of illicit drugs appeared to be a widespread practice among gay men in Melbourne. According to a 1998 survey by the ALSO Foundation (a gay charity) and the Australian Drug Foundation (Murnane, Smith, Crompton, Snow, \& Munro, 2000), two-thirds of gay men surveyed in Melbourne had used illicit drugs, with those under thirty years of age being the most likely to have used them. However, while such rates might suggest the existence of a somewhat relaxed attitude towards drug use, there are rules of comportment that ensure that drug use is well regulated. For example, there is a designated space for drugs that ensures drug use remains recreational and does not disrupt other aspects of lives. Illicit drugs might be accessible and acceptable for many gay men, but only particular drugs are accessible and acceptable, while others are not. With the former, there are also acceptable ways of administering these drugs, as well as acceptable activities engaged in while under their influence. There are both permissions and prohibitions designed to maximize the perceived benefits of using while minimizing the potential risks.

So, just as some men seem to have found the rule book for doing gay, gay ways of using structure drug use and gay men are expected to learn these rules. They do so from peers, including lovers, and in practice as a form of ethos or prevailing community disposition toward such drug use and all that accompanies it. This comprehension by our respondents of gay ways of using reveals how belonging to gay community is constituted in layers of expectation, including rules for behavior, a certain comportment exercised in the community's sociality, and living within an intricate web of relationality. It is not surprising that some might find these expectations difficult to meet, both psychologically and socially, and that they may have certain effects on drug-use practice as well. 
One effect of these community expectations might be that some gay men who come into contact with drugs within gay community are more likely to experiment with them. This is a result of having access to what are perceived to be safer ways of using drugs, for contact with a community of gay men experienced in drug use provides important information relating to administration and effects from the same men who supply the actual substances (see also Southgate \& Hopwood, 2001). Sharing such information becomes another thread in the web of relationality as gay men build their networks. This provision of lay information confers responsibility onto the drug user to look after himself, to avoid harm, not to have a negative impact on those sharing the experience, and not to bring unwanted attention to the gay male drug-using milieu through overdose or arrest. It also entails a responsibility to ensure that other participants in such drug-using events receive any support they need if encountering problems with their drugs. These responsibilities are designed to maximize group benefits while reducing the potential harms and dangers to individuals. If you want to be on the team, you must know and play by the rules. Therein rests the good gay citizen. Here the character of gay sociality emerges to choreograph and contextualize drug use, in spaces, times, and places particularly:

I think that it's a very accepted part of gay culture...I think there’s a definite separation between acceptable level of drug use and ways of using drugs. I think people are reasonably well informed of the effects of drugs, and I think the community tends to look after people quite well in terms of educating people who haven't used very much. Ah, in terms of looking after them, in terms of what the effects are, how to use them and minding them on those occasions. I think that's a really positive thing in the culture of drug use. I think that, ah, people tend to be quite constructive in their drug use. They're 
very knowing about it in terms of when they take it and what they’ve got coming up in the next week or whatever, and they modify their drug use accordingly, um. I think a lot of people have got very demanding careers and jobs.

Clearly, gay ways of using prevail, and a broader, more cultural notion of harm reduction could take significant advantage of this fact by addressing these ways. Yet, this does not constitute approval of such use at any significant level among Melbourne gay men; rather, it represents a conditional approval for proper use. And injection of drugs remains a marginal practice.

\section{The Particular Place of Drug Injection}

Beneath these gay ways of using, however, lurks the specter of problematic drug usage, which involves potentially negative personal and social effects. Functional use is okay; getting messy and, maybe, staying so are not:

Problem usage is when it starts impacting on the rest of their life, when it's not just something that they do because they want to. It's something they do because they have to and need to...it starts affecting their work and their social life and their friends and relationships, things like that. That's not good. That's not fun. That's not what most people do this stuff for. It's basically the difference between a habit and a hobby.

In this framing, habitual drug use attains a special status. Within Melbourne's gay men’s drug-using milieus, injecting drugs is regarded as emblematic of habitual usage, and a number of respondents noted that injecting is not an approved mode of using drugs and is one that gay ways of using described above are designed to prevent. As one respondent noted, “...despite the fact that drugs are so prevalent in 
our culture, there is still an enormous stigma about injecting drug use in the gay culture.”

There is other evidence from our study that supports such a claim. Thus far responses from study respondents with problematic patterns of drug using and with marked detachment from gay community have been less widely presented. Some of these men's lives were in fact distinctly messy or had been at various times in the past. Habitual injectors, particularly (but not only) heroin users, offered a sharp contrast to a constitutive story of gay community. Their stories told a different tale, and it is important to note that because the prevailing view of drug use within the gay community discounts the possibility of drug injection, those who do so are often left at a distance from potential community interventions to prevent HCV transmission. The discursive relegation of drug injection and problematic use to habit, with all its associated attributes of messiness and the underlying implication of addiction, has the tendency to individualize such use and therefore to construct the men who do inject as problematic. Such a construction is particularly easy to do when messy users use heroin rather that amphetamines and/or engage in sex work to support their habit, as was the case with a number of men in this study. The individuation that results focuses attention on the user as the problem and on fixing him, and it fits nicely within more conventional, psychosocial models of disease prevention and health promotion.

Much harm reduction is conceived in a similar way, with the goal of reducing the risk associated with using through provision to individuals of information and technical skills that minimize the potential transmission of both HIV and HCV. Yet a strong reliance on print resources and information about using (in Australia, reviewed in Dowsett et al., 1999) may not adequately address either the social forces and 
relational contexts that produce other ways of using (and possibly, within those ways, even drug of choice) or the processes of marginalization that effectively cut off some gay injectors from services and support because they do not conform to gay community expectations of drug use-that is, they get messy.

But not all gay men who inject do get messy, as a few respondents noted, and this offers a challenge to health promotion and prevention education:

I think there's a fairly high awareness in the injecting gay community of safer practices. And if the injector is recreational they are more likely to access equipment at times when [they] need it and therefore not be in a situation where they have to share a needle. They can actually plan ahead. Um, that could be a possibility. Ah, again, if someone, whether they're gay or not, if they need to use, they need to use and they may take the same risks whether they are gay or not. So, I would think the difference is between recreational user and the habitual user: in the habitual using population as we know the acquisition of Hep C [shorthand in Australia for hepatitis C] is very high. This indicates that reference to gay ways of using, if they were effectively utilized in education strategies, might be helpful for gay drug injectors as well, even if the community consensus does not currently understand and accept such use. However, this discursive weight of hobby within accepted gay ways of using and the discretion surrounding drug use generally, and particularly injecting, allow little room for tackling those issues and concerns comprising the notion of habit-that is, habit is unacceptable in gay community life so it cannot be addressed openly as a gay community issue. Thus, gay community initiatives geared to prevent hepatitis $\mathrm{C}$ and HIV transmission may neglect those men with the direst need by focusing mainly on 
those whom HIV/AIDS educators have always been good at reaching: men like themselves.

The Paradox of Gay Community Responses to Drug Injecting

This may not be the intent of community-based prevention efforts at all; but ironically, it might be those very gay ways of using that contribute to a less than optimal approach to unsafe drug use prevention. Indeed, in hepatitis C prevention, the perceptions of our respondents would indicate that gay community might be part of the problem. Some commentators argue that such social network embeddedness might actually inhibit risk reduction (Hughes, Heckathorn, Broadhead, Madray, \& van Hulst, 1998). Certainly, gay men’s community health agencies have largely been neglectful of drug-related harm, noticed most readily in relation to tobacco and alcohol use. Also, the camouflage surrounding gay men's drug use is exacerbated by the different gay communities’ standpoint within Australian HIV/AIDS politics, which calls for advocates and activists to protect their communities from homophobic and AIDS-related attacks. Questioning drug use in any specific community can be perceived as an attack on gay community as a whole, particularly as those best positioned to take up drug issues are themselves likely to have become gay men and a members of a gay community through the very forms of sociality and relationality described in this paper-including, possibly, its ways of using drugs. It is not, then, just drug use that becomes questioned, but also the very structure of the community gay men are making and the lives they live. For gay men who are community health service or education providers, and who have made a success of their lives as gay men living within a gay community, questioning drug use and thinking about it from the point of view of health may prove difficult to do. 
Gay community is not a determinant of kinds of behavior or activity here in any functionalist sense; rather, we need to see it as a structured field of ever-evolving practices, in which enactments can provide success, experience, solutions to sexual interests, real pleasures, and rewards. It is also a discursive field of expectations, ideas, desires, interpretations, and readings by gay men of each other, both socially and sexually. Our research respondents perceived that success on that field is neither guaranteed nor ever finally achieved — it has to be continually pursued. According to this view, no life memberships in gay community are available.

\section{Conclusion}

We have not dealt in this paper with many issues that arose in the study; these will be the subject of other papers, workshops, and presentations. Rather, we have been concerned here with the connections between gay community and drug use as seen by our respondents. We have shown that the sociality of gay community consists in, and is constitutive of, the practices of becoming and living as gay men as collective experience (rather than merely as individual behavior), and that it is essential to understand meanings and ideas about being and doing gay as they evolve. We have also shown that using drugs is one important aspect of that sociality. If men succeed in doing gay well, they learn to develop new kinds of relationships, not only sexual ones. The texture and quality of those relationships (i.e., relationality) include and constitute the ways in which drug using takes on a specific character, a particular function, and enhanced values. Shared drug experiences in part serve to constitute gay men's relationality in Melbourne in turn. This is also true for other drug-using subcultures; but in the case of gay communities, the alignment of sexual interests in a marginalized and stigmatized subculture with gay ways of using means that drug use becomes constitutive of a gay sexual subjectivity (Dowsett 1996b) as well. Therefore, 
the specificities and peculiarities of these gay communities need to be addressed in any attempts to insert hepatitis $\mathrm{C}$ prevention activities into a community already saturated with, and even defined by, HIV/AIDS prevention efforts. Such activities also need to address the particular articulations of being and doing gay, of living in or being marginalized from gay community, and of gay ways of using drugs.

The complexities of gay sociality and relationality provide both resources and dangers for gay men that seem both to increase the tendency for drug using and to provide a supportive, safe space for such use. They also encourage for many gay men intimate and/or communal and generally functional drug using as a matter of community comportment that fits within a web of mutually shared responsibility, although these outcomes cannot be guaranteed. These communities are resource rich in terms of explicit support and information processes-gay ways of using provide cultural resources and real avenues for prevention education and other kinds of interventions to stop the spread of HCV (and HIV). It is also clear, in Melbourne at least, that not all gay men can take advantage of what the gay community provides; that not all can abide by its rules or even comprehend them; and that some even see gay community as part of the problem, not as a resolution or safe resting place. Gay community attachment is a struggle for some men, and the contrasts between men in this study, specifically those enjoying gay community life and those feeling marginal to it, suggest that there are divergent effects and consequences of gay community on patterns of drug using, even including choice of drug. But no simple binaries suffice, whether on the basis of in or out of gay community, of use of heroin or speed, of communal or individual use, or even of habit or hobby pattern of use.

It will be important to comprehend the full complexity of what is occurring in gay communities in relation to sociality, relationality, sexuality, and drug use in order 
to develop effective health promotion programs related to hepatitis C. Such an approach may be very useful for studying other communities using drugs, particularly where there is another underlying social dynamic that defines that community over and above its drug use. For gay men, though, this study shows that it will be vital to utilize an understanding of gay ways of using more effectively in harm reduction programs and to recognize how social marginality works, how discretion operates to camouflage need, and how gay community educators are themselves implicated in the prevailing discourse on drug using, if hepatitis $\mathrm{C}$ is not to follow HIV in devastating another generation of gay men’s lives. 


\section{Acknowledgments}

The study was funded by the National Health and Medical Research Council, with additional support from the Australian National Council on HIV/AIDS and Related Diseases. We would like to acknowledge the support of VAC staff, particularly Daniel Madeddu and David Voon, who helped develop and undertake the study, and the project’s Reference Group-Carlo Campora, David Chong, Louise Compton, Chris Gill, Matt Dixon, Craig Mercer, and Kirsty Morgan—for its guidance and advice. We note with gratitude the terrific contribution of the study's research assistants, John Ryan and Kirk Peterson, and the support of the Australian Research Centre in Sex, Health \& Society at La Trobe University, Melbourne, as host institution. Particular thanks goes to Ann Mitchell and the Centre’s Community Education and Liaison Unit. Last but not least, we are deeply indebted to the study respondents who so readily told us their stories and let us delve into their lives. 


\section{References}

Agrosino, M. V., \& Mays de Pérez, K. A. (2000). Rethinking observation: from method to context. In N.K. Denzin \& Y.S. Lincoln, (Eds.), Handbook of Qualitative Research (2 ${ }^{\text {nd }}$ ed.), (pp. 673-702). Thousand Oaks, CA: Sage.

Benton, K.,W. K., \& Kirkby, R. J. (1994). Knowledge and practice of safe sex and alcohol and drug use in the Melbourne gay community. Venereology, 7(2), 969976.

Bollen, J., Edwards, B., Dowsett, G. W., McInnes, D., \& Couch, M. (1998). Understanding the contexts of risk-taking among Sydney gay men. (A Report to the New South Wales Department of Health, AIDS/Infectious Diseases Branch). Sydney, Australia: Macquarie University, Sociology Department.

Burawoy, M., Burton, A., Ferguson, A. A., Fox, K. J., Gamson, J., Gartrell. J., et al. (1991). Ethnography Unbound: Power and Resistance in the Modern Metropolis. Berkeley, CA: University of California Press.

Case, P., Clatts, M. C., Egan, J. E., \& Navaraez, R. F. (2000, July). HIV risks, resiliancy, and club drugs among men who have sex with men. Poster session to the XIII International AIDS Conference, Durban, South Africa.

Commonwealth of Australia (1989). National HIV/AIDS Strategy: A Policy Information Paper, Canberra, ACT: Australian Government Publishing Service. Commonwealth of Australia (1993). National HIV/AIDS Strategy, 1993-94 to 199596, Canberra, ACT: Australian Government Publishing Service.

Commonwealth of Australia (1996). "Partnerships in Practice”-National HIV/AIDS Strategy, 1996-97 to 1998-99, Canberra, ACT, Australian Government Publishing Service. 
Crofts, N., Louie, R., Rosenthal, D., \& Jolley, D. (1996). The first hit: circumstances surrounging initiation into injecting. Addiction, 91(8), 1187-1196.

Department of Human Services. (2002). Victorian Infectious Diseases Bulletin, 5(1) Melbourne, Australia.

Dowsett, G. W. (2003). Johnnie comes marching...where: Australian gay men, masculinity, HIV/AIDS and sex. Culture, Health and Sexuality, 5(3), 237-248.

Dowsett, G.W. (1996a). Perspectives in Australian HIV/AIDS health promotion. In NSW AIDS/Infectious Diseases Branch (comp.), NSW HIV/AIDS Health Promotion Conference: Keynote Addresses, Selected Papers and Future Directions , (pp.19-31). Sydney, Australia: New South Wales Health Publication (AIDS) 96-0067.

Dowsett, G. W. (1996b). Practicing desire: Homosexual sex in the era of AIDS. Stanford University Press: Stanford, CA.

Dowsett, G. W. (1993). Sustaining safe sex: sexual practices, HIV and social context. AIDS 92/93, 7(suppl 1), S257-262.

Dowsett, G. W., Bollen, J., McInnes, D., Couch, M., \& Edwards, B. (2001). HIV/AIDS and constructs of gay community: researching educational practice within community-based health promotion for gay men. International Journal of Social Research Methodology, 4(3), 205-223.

Dowsett, G. W. \& McInnes, D. (1996a, July). “Post-AIDS”: assessing the long-term social impact of HIV/AIDS in gay communities. Vancouver, Canada: paper presented at the XI International Conference on AIDS.

Dowsett, G. W. \& McInnes, D. (1996b). Gay community, AIDS agencies and the HIV epidemic in Adelaide: theorising “post-AIDS”. Social Alternatives, 15(4), 29-32. 
Dowsett, G. W., Misson, S., McDonnell, E., \& McInnes, D. (2001). The training needs of the community-based HIV/Hepatitis C education workforce (CEWT Broadsheet \#5). Melbourne, Australia, Australian Reseach Centre in Sex, Health and Society, La Trobe University.

Dowsett, G. W., Turney, L., Woolcock, G., Rance, A., \& Thomson, N. (1999).

Hepatitis $C$ prevention education for injecting drug users in Australia: A research report. Canberra, Australia: Commonwealth Department of Health and Aged Care. Available online at:

http://www.health.gov.au/pubhlth/publicat/document/hepc_idu.pdf

Grierson, J. \& Misson, S. (2002). HIV futures 3 regional reports: Victoria.

Melbourne, Australia: La Trobe University, Australian Research Centre in Sex, Health and Society.

Grierson, J., Misson, S., McDonald, K., Pitts, M., \& O’Brien, M. (2002). HIV futures

3: Positive Australians on services, health and well-being. Melbourne, Australia: La Trobe University, Australian Research Centre in Sex, Health and Society.

Hughes, J., Heckathorn, D. D., Broadhead, R. S., Madray, H., \& van Hulst, Y. (1998, June/July). Injector network embeddedness inhibits the effectivness of risk reduction efforts. Geneva, Switzerland: poster session at the XII International Conference on AIDS.

Hurley, M. \& Dowsett, G. (1994). Songs my lovers taught me. National AIDS Bulletin, 8(10/11), 22-23.

Ireland, K., Knox, S., Van de Ven, P., Howard, J., \& Kippax, S. (1999). Using and "the scene": Patterns and contexts of drug use among Sydney gay men. Sydney, 
Australia: The University of New South Wales, National Centre in HIV Social Research.

Kippax, S., Connell, R. W., Dowsett, G. W., \& Crawford, J. (1993). Sustaining safe sex: Gay communities respond to AIDS. London, UK: Falmer Press.

Maher, S. (1999, November 9). Coming down. Sydney Star Observer, p. 13.

Murnane, A., Smith, A., Crompton, L., Snow, P., \& Munro, G. (2000). Beyond Perceptions: A report on alcohol and other drug use among gay, lesbian and queer communities in Victoria. Melbourne, Australia: ALSO Foundation and the Australian Drug Foundation Centre for Youth Drug Studies.

Myers, T., Aguinaldo, J., Bullock, S., Leaver, C., Dakars, D., Calzavara, L., et al. [n.d.]. Desired effects - sex or drugs? Gay and bisexual men who use injection and non injection drugs: preferences, experience and role of drugs in unsafe sex. Toronto, Canada: University of Toronto, HIV Social, Behavioural and Epidemilogical Studies Unit, personal communication-powerpoint presentation. National Centre in HIV Epidemiology and Clinical Research. (2004). Australian HIV Surveillance report, 20(2).

National Centre in HIV Epidemiology and Clinical Research (Ed.). (2002). HIV/AIDS, viral hepatitis \& sexually transmissable infections in Australia annual surveillance report 2002. Sydney, Australia: The University of New South Wales.

National Centre in HIV Social Research (Eds) (2003). HIV/AIDS, hepatitis C \& related diseases in Australia: Annual report of behaviour 2003. Sydney, Australia: The University of New South Wales. 
National Lesbian and Gay Health Association. (1998, July). Gay male HIV prevention work in a "post-AIDS" era. Pre-conference institute conducted at the National Lesbian and Gay Health Conference, San Francisco, CA.

Plummer, K. (1995). Telling sexual stories: Power, change and social worlds. London, UK: Routledge.

Prestage, G., Knox, S., Kippax, S., Benton, K., Mahat, M., Crawford, J., et al. (1997). A demographic and behavioural comparison of three samples of homosexually active men in Sydney, Melbourne and Brisbane. Sydney, Australia: The University of New South Wales, National Centre in HIV Social Research.

Race, K. D. (2003). Revaluation of risk among gay men. AIDS Education and Prevention, 15, 369-381.

Rhodes, F., Carlson, R. G., Cottler, L., Stark, M. J., Lambert, E. Y., \& Trotter, R. L. (1998, June/July). Understanding HIV risks of drug-using men who have sex with men. Geneva, Switzerland: Poster session at the XII Interntional Conference on AIDS.

Richters, J., Van de Ven, P., Knox, S., Prestage, G., Crawford, J., \& Kippax, S. (1997)._Sydney gay community surveillance report no.5: Update to June 1997. Sydney, Australia: The University of New South Wales, National Centre in HIV Social Research.

Rofes, E. (1998). Dry bones breathe: Gay men creating post-AIDS identities and cultures. Binghamton, NY: Haworth Press.

Sharp, R., Crofts, N., Sattler, G., Marcus, L., Meade, J., Wallace, J., et al. (1996). Negotiated safety? Sexual practices among young gay injecting drug users. Venereology, 9(2), 106-112. 
Sharp, R., Davis, M., Dowsett, G. W., Kippax, S., Hewitt, K., Morgan, S., et al. (1991). Ways of using: Functional injecting drug users project. Sydney, Australia: Macquarie University, Centre for Applied Social Research.

Southgate, E. \& Hopwood, M.. (1999). The drug use and gay men project: Issues papers, July 1999. Sydney, Australia: The University of New South Wales, National Centre in HIV Social Research.

Southgate, E. \& Hopwood, M. (2001). The role of folk pharmacology and lay experts in harm reduction: Sydney gay drug using networks. The Intenational Journal of Drug Policy, 12, 321-335.

Strauss, A. \& Corbin, J.. (1990). Basics of qualitative research. Newbury Park, CA: Sage.

Watney, S. (1999). Safer sex as community practice. In R. Parker \& P. Aggleton (Eds), Culture, society and sexuality: A reader (pp.405-415). London, UK: University College London Press. 


\section{Author Note}

Correspondence concerning this article should be addressed to Gary W. Dowsett, Australian Research Centre in Sex, Health and Society, La Trobe University, $1^{\text {st }}$ floor, 215 Franklin Street, Melbourne, VIC 3000, Australia. E-mail:

g.dowsett@latrobe.edu.au; David Wain, Australian Research Centre in Sex, Health and Society, La Trobe University, $1^{\text {st }}$ floor, 215 Franklin Street, Melbourne, VIC 3000, Australia. E-mail: d.wain@latrobe.edu.au; Deborah Keys, The Key Centre for Women's Health in Society,

The University of Melbourne, Victoria 3010, Australia. E-mail: keysdw@unimelb.edu.au 


\section{University Library}

\section{- M M I N E R VA A gateway to Melbourne's research publications}

Minerva Access is the Institutional Repository of The University of Melbourne

Author/s:

Dowsett, Gary W.;Wain, David;Keys, Deborah

Title:

Good gay men don't get "messy": injecting drug use and gay community

Date:

2005

Citation:

Dowsett, G. W., Wain, D., \& Keys, D. (2005). Good gay men don't get "messy": injecting drug use and gay community. Sexuality Research and Social Policy: Journal of NSRC, 2(1), 22-36.

Publication Status:

Published

Persistent Link:

http://hdl.handle.net/11343/34390 